\title{
Frequent gain- and loss-of-function mutations of the BjMYB113 gene accounted for leaf color variation in Brassica juncea
}

\author{
Guanghui An and Jiongjiong Chen ${ }^{*}$ (D)
}

\begin{abstract}
Background: Mustard (Brassica juncea) is an important economic vegetable, and some cultivars have purple leaves and accumulate more anthocyanins than the green. The genetic and evolution of purple trait in mustard has not been well studied.

Result: In this study, free-hand sections and metabolomics showed that the purple leaves of mustard accumulated more anthocyanins than green ones. The gene controlling purple leaves in mustard, Mustard Purple Leaves (MPL), was genetically mapped and a MYB113-like homolog was identified as the candidate gene. We identified three alleles of the MYB113-like gene, BjMYB113a from a purple cultivar, BjMYB113b and BjMYB113c from green cultivars. A total of 45 single nucleotide polymorphisms (SNPs) and $8 \mathrm{lnDels}$ were found between the promoter sequences of the purple allele BjMYB113a and the green allele BjMYB113b. On the other hand, the only sequence variation between the purple allele BjMYB113a and the green allele BjMYB113C is an insertion of 1,033-bp fragment in the 3'region of BjMYB113c. Transgenic assay and promoter activity studies showed that the polymorphism in the promoter region was responsible for the up-regulation of the purple allele BjMYB113a and high accumulation of anthocyanin in the purple cultivar. The up-regulation of BjMYB113a increased the expression of genes in the anthocyanin biosynthesis pathway including BjCHS, BjF3H, BjF3'H, BjDFR, BjANS and BjUGFT, and consequently led to high accumulation of anthocyanin. However, the up-regulation of BjMYB113 was compromised by the insertion of 1,033-bp in 3'region of the allele BjMYB113c.
\end{abstract}

Conclusions: Our results contribute to a better understanding of the genetics and evolution of the BjMYB113 gene controlling purple leaves and provide useful information for further breeding programs of mustard.

Keywords: Leaf color, MYB transcription factor, BSR-seq, Map-based cloning, Brassica juncea

\section{Background}

Mustard (Brassica juncea, $2 \mathrm{n}=36, \mathrm{AABB}$ ) is an important economic vegetable in the world $[1,2]$. This cultivated species contains several different varieties, which exhibit extreme morphologic polymorphisms, with leaves, stems, roots, or seeds as harvest organs. For leafy

\footnotetext{
* Correspondence: jjchen@mail.hzau.edu.cn

Key Laboratory of Horticultural Plant Biology, Ministry of Education, Key Laboratory of Horticultural Crop Biology and Genetic Improvement (Central Region), MOA, College of Horticulture and Forestry Sciences, Huazhong Agricultural University, 430070 Wuhan, People's Republic of China
}

mustard cultivars, some have green leaves while others have purple leaves. The purple color is caused by the accumulation of anthocyanins in leaf epidermal cells [3].

Anthocyanins are a large group of water-soluble natural pigments and widely distribute in higher plants. They are largely responsible for the red, purple, and blue colors in flowers, fruits, leaves, seeds and other plant organs $[4,5]$. In plants, anthocyanins play important roles in attracting pollinators and seed distributors [5]. Anthocyanins can protect plants from UV damage, and they have strong capacity to scavenge reactive oxygen species

\section{$\triangle B M C$}

(c) The Author(s). 2021 Open Access This article is licensed under a Creative Commons Attribution 4.0 International License, which permits use, sharing, adaptation, distribution and reproduction in any medium or format, as long as you give appropriate credit to the original author(s) and the source, provide a link to the Creative Commons licence, and indicate if changes were made. The images or other third party material in this article are included in the article's Creative Commons licence, unless indicated otherwise in a credit line to the material. If material is not included in the article's Creative Commons licence and your intended use is not permitted by statutory regulation or exceeds the permitted use, you will need to obtain permission directly from the copyright holder. To view a copy of this licence, visit http://creativecommons.org/licenses/by/4.0/ The Creative Commons Public Domain Dedication waiver (http://creativecommons.org/publicdomain/zero/1.0/) applies to the data made available in this article, unless otherwise stated in a credit line to the data. 
(ROS), which is critical for biotic and abiotic tolerance [6-11]. This group of metabolites also promotes human health with obvious functions against obesity, inflammation, coronary arteriosclerosis, and cancers [12-16].

Many previous studies have shown that transcriptional regulation of anthocyanin biosynthesis in higher plants is mainly controlled by the MBW ternary complex containing MYB, bHLH and WD repeat transcription factors $[17,18]$. For example, AtMYB113 forms a complex with a bHLH and a WD40 protein, which actives the genes in the anthocyanin biosynthetic pathway leading to pigment accumulation in Arabidopsis thaliana [19]. The MBW ternary complex transcriptionally regulates the genes in the anthocyanin biosynthetic pathway, including phenylalanine-ammonia lyase (PAL), chalcone synthase (CHS), chalcone isomerase (CHI), flavanone 3hydroxylase $(F 3 H)$, dihydroflavonol 4-reductase (DFR), and anthocyanidin synthase (ANS) [20,21]. Upregulation of components of the MBW complex has been widely researched and shown to be responsible for high anthocyanin accumulation in many plant species such as peach [22], apple [23], pear [24], grape [25], blood orange [26], petunia [27], rice [28], lettuce [29], bokchoy [30], kale [31], cauliflower [32], barrel medic [33], etc. In addition to the MBW complex, other transcription factors, such as NACs, HY5, ERFs, BBX22, and WRKY, were found to be involved in anthocyanin biosynthesis as well. These transcription factors can bind to the promoters of component genes of the MBW complex indirectly or directly to regulate their expressions and consequently anthocyanin biosynthesis [22, 34, 35].

The genus Brassica harbors several important vegetable crops, such as cabbage, cauliflower, Chinese cabbage, and mustard, all with both green and purple cultivars. Purple is an attracting color in vegetables, and its underlying metabolites provide health benefits to consumers. In Brassica rapa, mutants of the anl locus and the Anp locus failed to produce anthocyanin pigments; BrEGL3.1 and BrEGL3.2, two genes encoding bHLH transcription factors, promote anthocyanin biosynthesis in Chinese cabbage. The activated BOMYB2 gene accounts for the anthocyanin accumulation in purple cabbage and cauliflower [31, 32]. Recently, a genetic analysis of the purple trait in mustard using an $F_{2}$ population derived from a cross between a purple and a green cultivar suggested that an insertion in the first intron of a MYB-encoding gene (BjPur) changed the purple leaves to green [3]. However, the function of the BjPur gene in mustard has not been verified, and the genetic and molecular mechanisms underlying purple mustard remain unclear.

Compared with wild progenitors, cultivated horticultural crops showed rich color polymorphisms. The gain-of-function mutations on the anthocyanin pathway have mainly occurred in the encoding genes of members of the MBW complex (see above). For example, the BoMYB2 gene in Brassica oleracea was activated independently for at least three times causing purple color in kale, cauliflower, and cabbage. The activation events in BoMYB2 included an insertion of a CACTA transposon, an insertion of a harbinger transposon and point mutations in its promoter region, respectively [31, 32]. Four genes encoding transcription factors bHLH, R2R3-MYB, R3-MYB and WD40, respectively, showed sequence and functional polymorphisms in cultivated lettuce, and mutations on three of them promoted accumulation of anthocyanins on lettuce leaves [29].

In this study, we dissected the genetics controlling purple mustard using Bulked Segregant Analysis and RNA-seq (BSR-seq). The gene was genetically finemapped and functionally confirmed through agrobacteria-mediated transformation. The mutation events were analyzed in detail, and the evolution of the causal gene was proposed. Our study contributes to a better understanding of the genetics and evolution of the gene controlling purple leaves and provides useful information for further breeding programs of mustard.

\section{Results}

\section{Characterization of anthocyanin in mustard cultivars}

Mustard has both green and purple cultivars (Fig. 1a, b, c). We compared the total anthocyanin contents of one purple mustard cultivar (pl102) and two green cultivars (rt104 and gre101). As expected, the total anthocyanin content in the purple cultivar was considerably higher than those in the two green cultivars (Fig. 2a). Free-hand sections of leaf tissues of the purple cultivar showed that the anthocyanins accumulated mainly in the epidermal cells and in less extent in the mesophyll cells near the epidermises. In contrast, no obvious anthocyanins were found in green cultivars (Fig. 1d).

We further used Liquid Chromatography-Electrospray Ionization-Mass Spectrometry (LC-ESI-MS) to analyze the types of anthocyanins and their concentrations in the purple and green cultivars. A total of 18 anthocyanins were identified (Table S1). Of them, six had significantly higher concentration in the purple cultivar than in green one $(p<0.05)$, including Delphinidin $3,5-\mathrm{O}$ diglucoside, Delphinidin 3-O-(6"-O-malonyl)-beta-Dglucoside, Cyanidin 3-O-(6"-O-malonyl)-beta-D-glucoside, Pelargonidin 3-O-glucoside, Peonidin 3,5-O-diglucoside, and Peonidin 3-O-glucoside, all emitted dark color (Fig. 2b). We conclude that the purple color in mustard leaves was caused by high accumulation of these anthocyanins. 


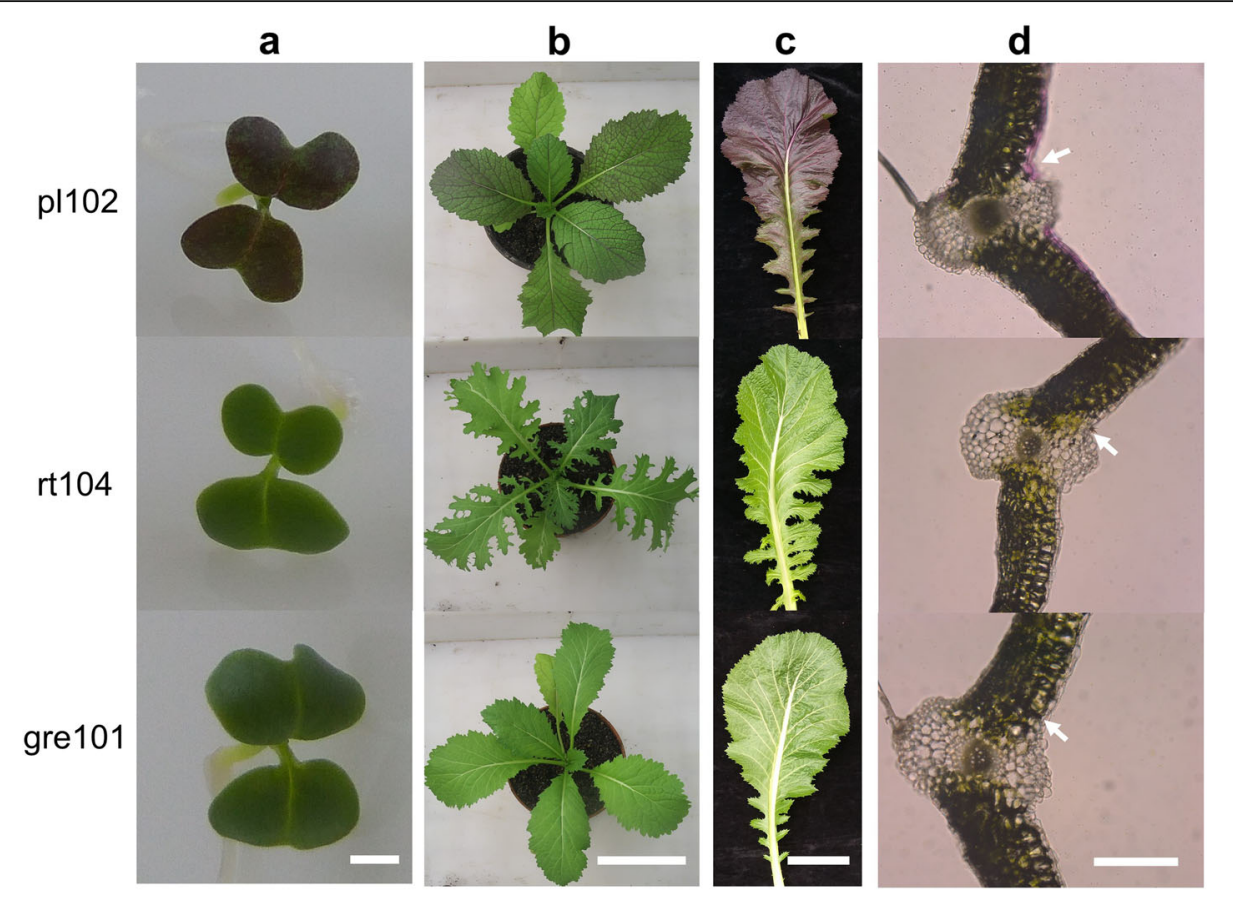

Fig. 1 Color variation in three Brassica juncea cultivars, pl102, rt104, gre101. a Four-day-old seedlings for pl102, rt104 and gre101. Scale bar represents $1 \mathrm{~mm}$. b One-month-old seedlings for pl102, rt104 and gre101. Scale bar represents $10 \mathrm{~cm}$. c Three-month-old leaves for pl102, rt104 and gre101. Scale bar represents $10 \mathrm{~cm}$. d Free-hand sections of leaf vein tissues for pl102, rt104 and gre101. The white arrows indicate that anthocyanins accumulated in the epidermal cells of purple leaves of pl102, but were absent in rt104 and gre101. Scale bar represents $0.1 \mathrm{~mm}$

\section{Map-based cloning of the gene controlling color variation in mustard}

As mentioned in Background section, a previous study suggested that an insertion of 1,268-bp fragment in the first intron of a $M Y B$ gene was responsible for its loss-of-function and green leaves [3]. Furthermore, some green cultivars such as rt104 and gre101 do not have the 1,268-bp insertion, and therefore should have distinct mechanisms for loss-of-function. To answer these questions, purple cultivar pl102 was crossed with green cultivars rt104 and gre101, respectively. Both $\mathrm{F}_{1}$ hybrids were purple, and they were selfed to generate two $\mathrm{F}_{2}$ segregating populations.

Of the 137 individuals in the $F_{2}$ segregating population derived from the cross pl102 $\times$ rt104, 102 individuals had purple leaves and 35 individuals had green leaves, with an expected ratio of $3: 1\left(\chi^{2}=0.0219<\chi^{2}(0.05,1)=3.84, P>\right.$ 0.05 , Table S2), suggesting a single gene controlling the color variation, which was referred to as Mustard Purple Leaves $(M P L)$ in this study. Then, we used BSR-seq to genetically map the gene underlying the color variation. The MPL gene was mapped to chromosome J15 (B05) (Fig. 3a). Screening a total of 1,353 individuals from the $F_{2}$ population ultimately mapped the $M P L$ gene between markers AGH260 and AGH263 on J15 (B05), in an interval of approximately $170 \mathrm{~kb}$ region (Fig. 3b, c). Only 12 open reading frames (ORFs) were predicted in this interval. The $M Y B$ gene, which is an ortholog of MYB113 in Arabidopsis and was previously shown to control color variation in mustard, was also located in the candidate interval and remained a reliable candidate gene (BjMYB113) (Fig. 3d). As mentioned above, the 1,268-bp insertion, which was suggested to inactivate the candidate gene in a previous study, was absent in the green parent [3]. To verify the $B j M Y B 113$ as the candidate gene, we first investigated its sequence variation between the two parents, including its 2 , 842 bp upstream sequences. A total of 45 SNPs and 8 InDels were found between the two alleles from the purple parent pl102 and the green parent rt104 (Fig. 4).

Interestingly, such sequence variation was not found between the purple parent pll02 and another green cultivar gre101. The BjMYB113 gene from green cultivar gre101 is almost identical to that from the purple cultivar, with only one SNP at $-2,269$ of promoter region (Fig. 4), and the BjMYB113 gene from either cultivar does not have the 1,268-bp insertion in intron 1 . BSRseq analysis of the pl102 $\times$ gre101 segregating population also showed that the gene controlling leaf color was located in the same region as BjMYB113 on chromosome J15 (Fig. S1) (see above). We then sequenced its flanking region and discovered an insertion of 1,033-bp fragment at 2,873 bp downstream its stop codon. Therefore, there are at least four alleles of the BjMYB113 gene, including BjMYB113a (purple allele), BjMYB113b (green allele) and $B j M Y B 113 c$ (green allele with the 1,033-bp 

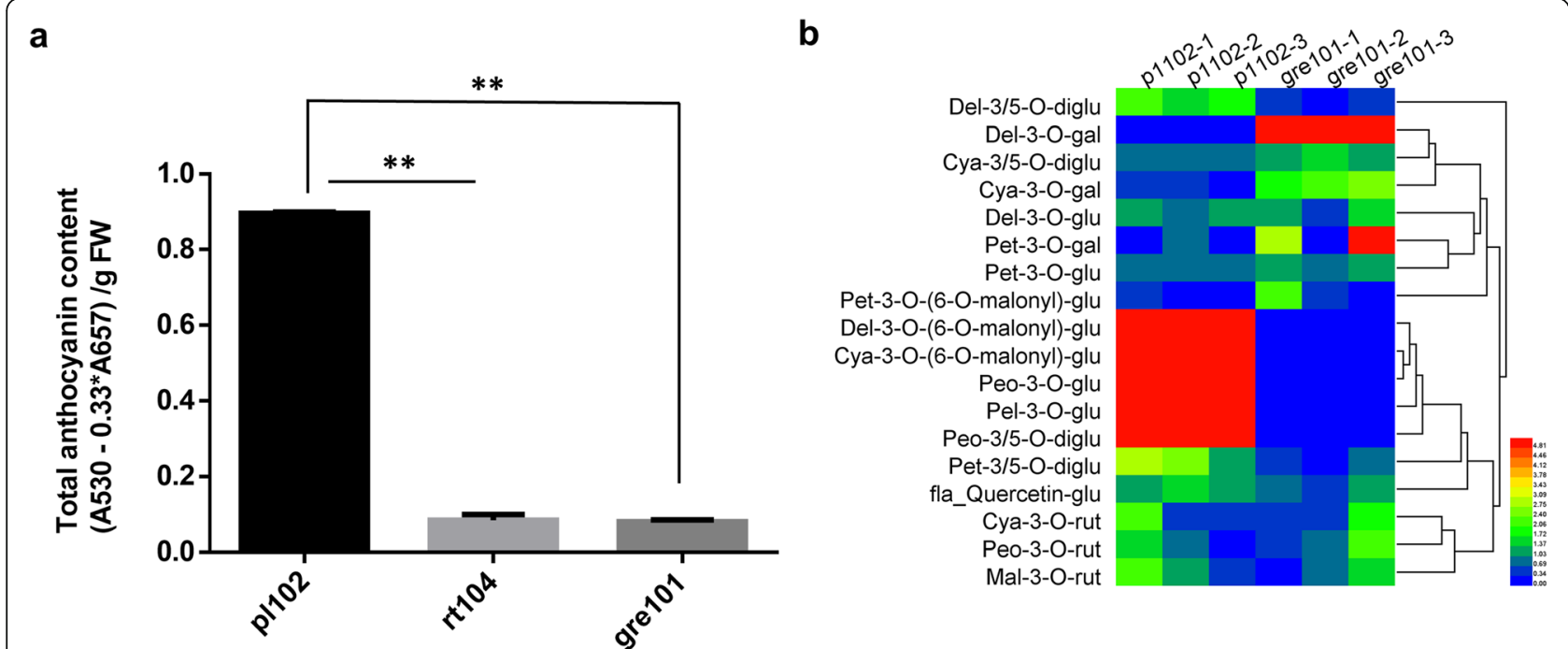

Fig. 2 Analysis of total anthocyanin contents and anthocyanin compounds in purple and green parental mustard cultivars. a Total anthocyanin contents of purple and green cultivars (leaves). b Heatmap of anthocyanin compounds in purple (pl102) and green (gre101) cultivars with three biological replicates, respectively. The abbreviations of anthocyanin compounds were shown in the left, and the corresponding full name of anthocyanin compounds were list in the Table S1. Statistical analyzes were conducted by Student's $t$ test, ${ }^{* *} P<0.01$

insertion in its 3' downstream), as well as an allele identified previously with an insertion of 1,268-bp fragment in the first intron (Fig. 4).

\section{Overexpressing the BjMYB113a, BjMYB113b, or BjMYB113c} alleles promoted anthocyanin accumulation in

\section{Arabidopsis}

The coding sequence of the three alleles BjMYB113a, $B j M Y B 113 b$ and $B j M Y B 113 c$ are highly conserved in different mustard cultivars (Fig. S2). The coding sequence of BjMYB113a and BjMYB113c are identical, while $B j M Y B 113 a$ and $B j M Y B 113 b$ vary by three SNPs, with only one of them leading to amino acid change (M120K) (Fig. S2). To verify the function of these three BjMYB113 alleles, their coding sequence were driven by cauliflower mosaic virus (CaMV) $35 \mathrm{~S}$ promoter and transferred into A. thaliana Col-0. All Arabidopsis lines overexpressing BjMYB113 alleles had purple leaves and stems in comparison with green leaves and stems in wild type (Fig. 5c, d, e, f). As expected, the color change was due to high accumulation of anthocyanins and high expression of BjMYB113 (Fig. 5a, b). Above results indicated that the proteins encoded by the three BjMYB113 alleles were functionally identical. Therefore, the color variations of mustard cultivars were most likely caused by expression difference of the BjMYB113 alleles.

Promoter region but not coding region is responsible for the activation of the BjMYB113a gene

We then investigated the expression level of the BjMYB113 gene in different mustard cultivars. A quantitative real-time PCR analysis demonstrated that the expression level of BjMYB113a (from purple cultivar) was significantly higher than $B j M Y B 113 b$ and $B j M Y B 113 c$ (from green cultivars) (Fig. 6a). We hypothesize that the expression difference between alleles BjMYB113a and BjMYB113b was caused by their promoter regions, and the expression difference between alleles BjMYB113a and BjMYB113c was caused by the 1,033-bp insertion in downstream region of the latter.

To test above hypothesis, promoter activity of the three BjMYB113 alleles was analyzed. GUS gene driven by the promoter of BjMYB113a, BjMYB113b, and $B j M Y B 113 c$, respectively, was transformed into Arabidopsis Col-0. Six transgenic lines were obtained for each allele, and the GUS activities of these transgenic lines were measured. The GUS activity driven by promoter of BjMYB113a or BjMYB113c was significantly higher than that driven by the promoter of $B j M Y B 113 b$, but no activity difference was observed between the promoters of BjMYB113a and BjMYB113c (Fig. 6b). Therefore, the polymorphisms in the promoter region accounts for the expression difference between BjMYB113a and BjMYB113b, and the low expression of $B j M Y B 113 c$ was likely caused by the 1,033-bp insertion in its downstream rather than the point mutation in its promoter region.

Forty-five SNPs and eight InDels were found between the promoter regions of BjMYB113a and $B j M Y B 113 b$. The motif analysis was carried out for these two promoters. The activation of the BjMYB113a in purple cultivar pl102 was likely due to the point mutations at $-1,317$ ( $\mathrm{C}$ to $\mathrm{G}$ ), $-1,576$ (A to 


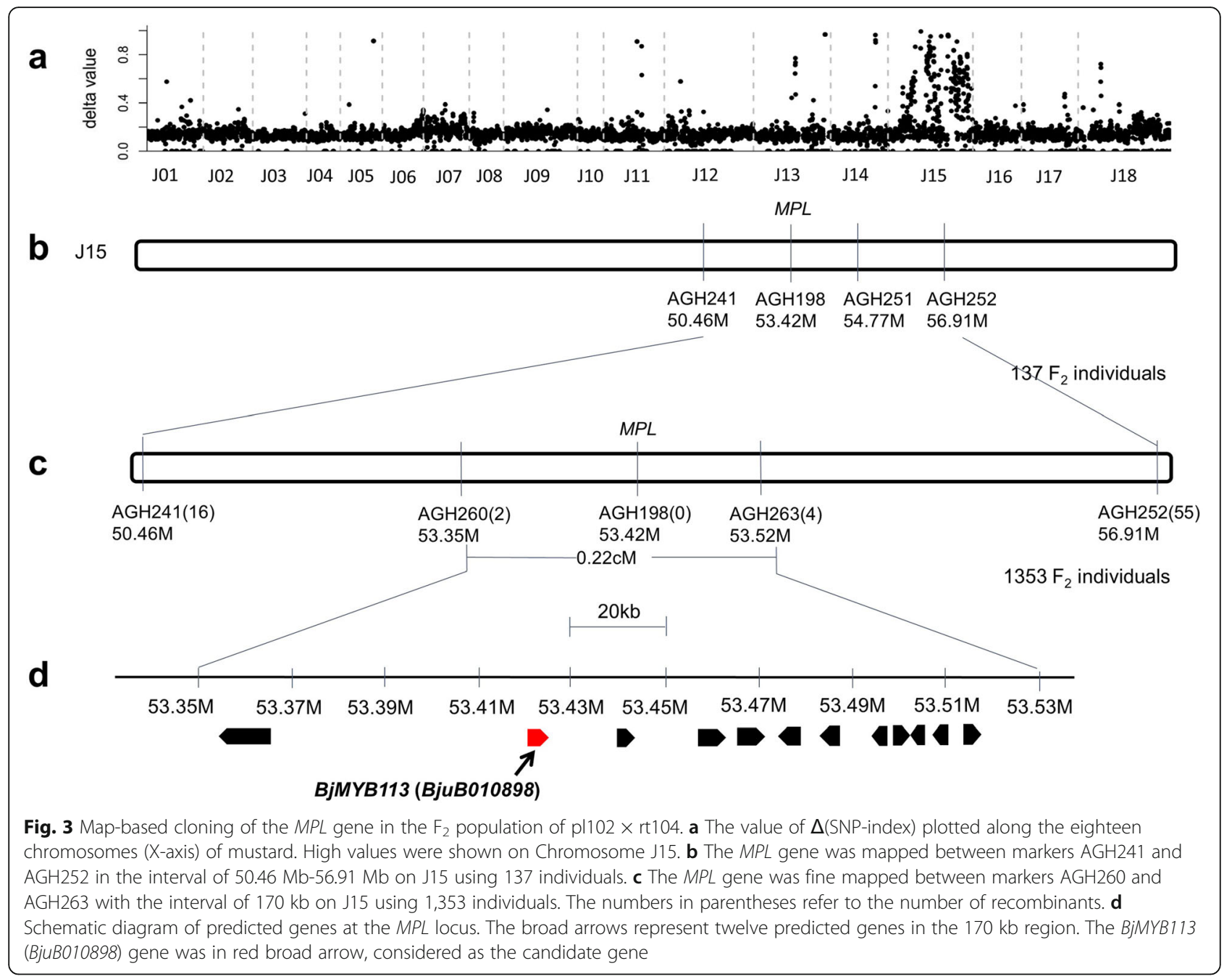

G), $-1,588$ (G to T), $-2,593$ (C to T). These four SNPs produced new transcription factor binding sites, including a MBSI motif (AAAAAAC(G/C)GTTA ), a bHLH motif (CANNTG), and two TATA-box motifs (ATTATA).
The 1,033-bp insertion suppresses the expression of BjMYB113C

To verify the effects of the 1,033-bp insertion on the expression of $B j M Y B 113$, the BjMYB113c allele, including its native promoter and 3'region was transformed to

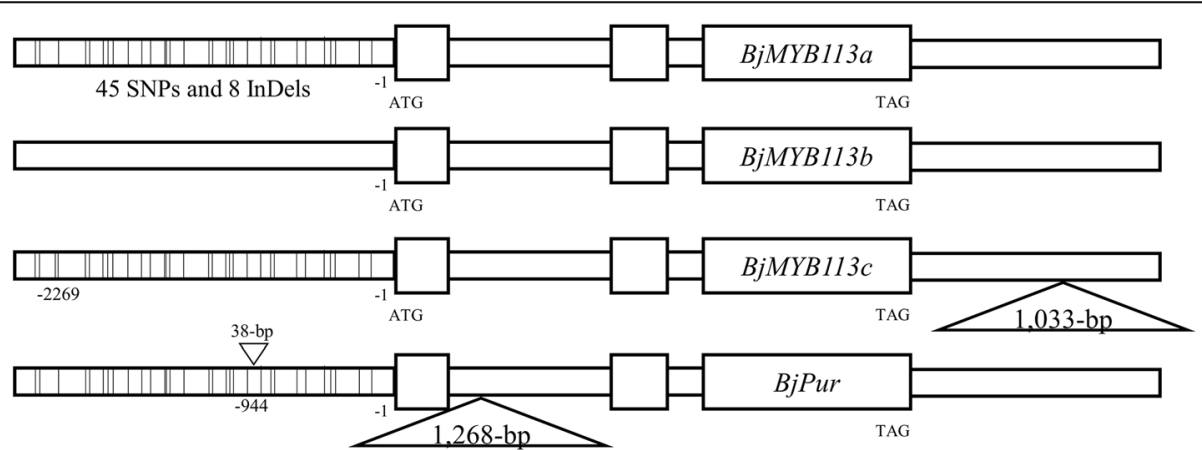

Fig. 4 The variations of four BjMYB113 alleles. The vertical lines in promotor region represent point mutations and InDels. The triangle in 3'region or intron represents fragment insertion 

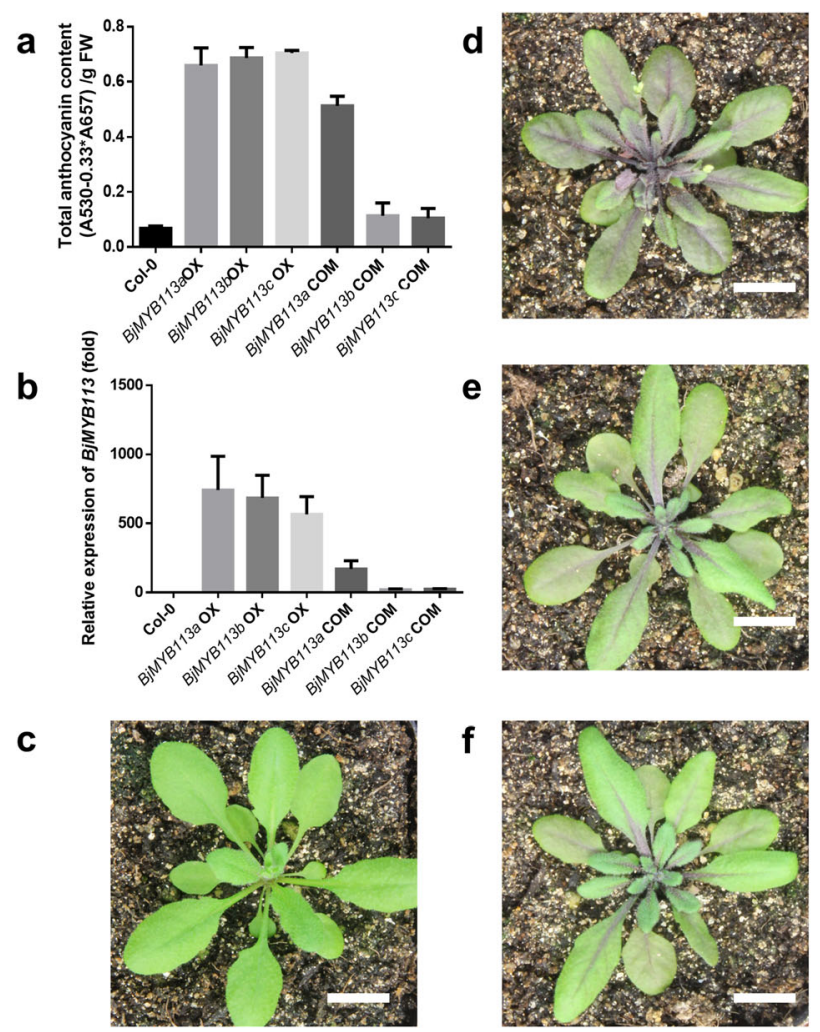

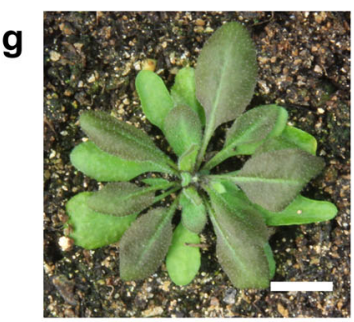

h
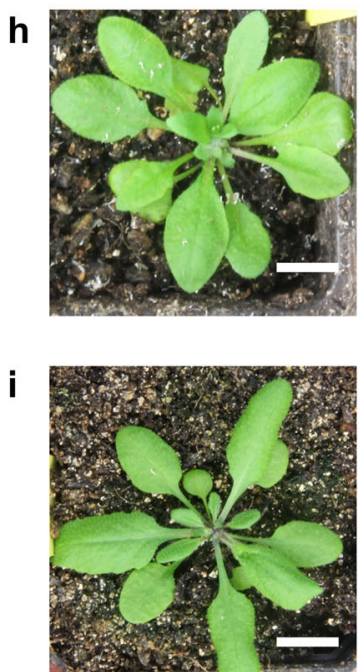

Fig. 5 The total anthocyanin content and the expression of BMMYB113 in Arabidopsis transgenic lines. a The total anthocyanin content in Arabidopsis transgenic lines. Col-0 is green wild-type Arabidopsis as the control, "BjMYB113a OX" refers to overexpression transgenic lines of BjMYB113a (CDS), "BJMYB113a COM" refers to complementary transgenic line of BjMYB113a (with native promotor and 3'region sequence). $\mathbf{b}$ The expression of BjMYB113 in the Arabidopsis transgenic lines. c The wild-type Arabidopsis Col-0. d-f BjMYB113a (CDS), BjMYB113b (CDS) and BjMYB113C (CDS) overexpression transgenic lines. g-i BjMYB113a, BjMYB113b and BjMYB113c complementary transgenic line. Scale bar represents $1 \mathrm{~cm}$

Arabidopsis Col-0. No color change was observed in positive transgenic lines (Fig. 5i). In contrast, transgenic line of a construct containing BjMYB113a gene with its native promoter and 3 'downstream sequences did change the color from green to purple (Fig. 5g). Note the main sequence difference between above two constructs was the 1,033-bp insertion. We conclude that high expression of BjMYB113a was due to mutations in its promoter region, and that the low expression of BjMYB113c was caused by the suppression effects of the 1,033-bp fragment inserted in its downstream region.
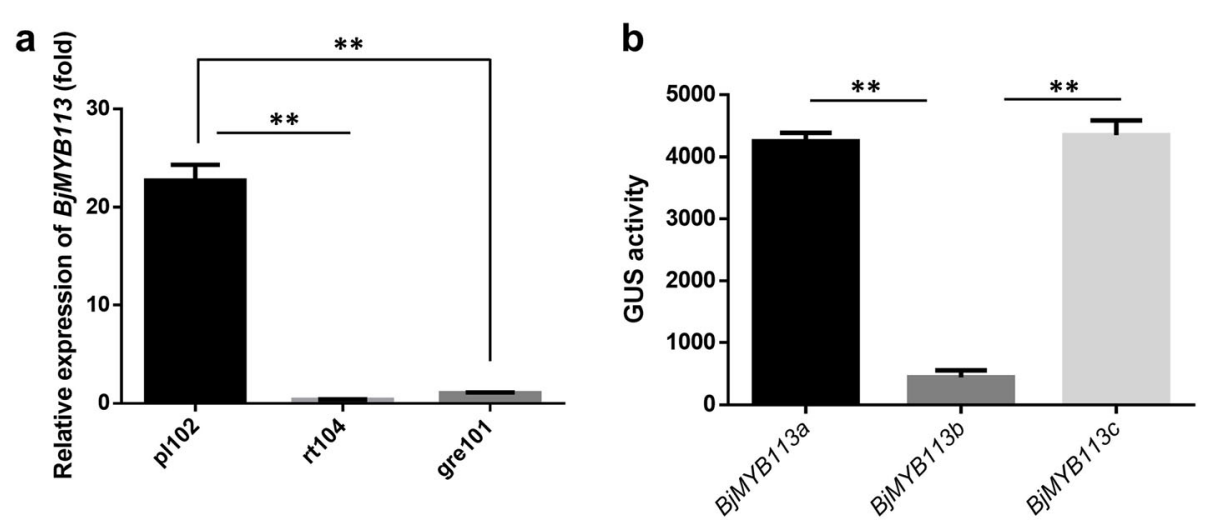

Fig. 6 a The relative expression of BjMYB113 in pl102, rt104 and gre101. b The promotor activity of BjMYB113a, BjMYB113b and BjMYB113C. Statistical analyzes were conducted by Student's t test, ${ }^{* *} P<0.01$ 
High expression of the BjMYB113a activated the anthocyanin biosynthetic genes

As an important component in the MBW transcription factor complex, activated BjMYB113 gene may up-regulate multiple genes in the anthocyanin biosynthesis pathway. qRT-PCR was carried out to investigate the expression difference of genes, including BjPAL (BjuA036480), BjC4H (BjuB015902), BjCHS (BjuA041225), BjCHI (BjuA004576), BjF3H (ВjuA035478), ВjF3'H (ВjuA047311), BjDFR (BjuA033678), BjANS (BjuB044852), and BjUGFT (BjuA047199). The qRT-PCR results showed that the expression of BjCHS, BjF3H, BjF3'H, BjDFR, BjANS, and $B j U G F T$ in purple cultivar pl102 were significantly higher than that in green cultivars rt104 and gre101 (Fig. 7).

\section{The evolution of the BjMYB113 gene}

To understand the evolution of the BjMYB113 gene in mustard, approximately $3 \mathrm{~kb}$ promoter sequences of 58 mustard accessions including 5 purple and 53 green ones were PCR amplified and sequenced. All accessions were also genotyped for the 1,268-bp insertion in intron 1 and the 1,033-bp insertion in its downstream. A neighbor-joining tree was constructed using the fulllength gene sequences, with BoMYB113 from Brassica oleracea as an outgroup (Fig. 8). Two major clades were found for these gene sequences from mustard, varying at least 50 nucleotides in promoter sequences between members from the two clades. The six sequences from Clade 2 are identical, all with the inactivated promoter as in green cultivar rt101 (BjMYB113b). The Clade 1 contains five distinct sequences including the sequence of $B j M Y B 113 a$, which has the activated promoter and lacks the 1,033-bp insertion in its downstream. The second sequence (with three cultivars) is BjMYB113c with one nucleotide different from $B j M Y B 113 a$ in promoter sequence and has the 1,033-bp insertion in its downstream. The third sequence (two cultivars) varies only two nucleotides from BjMYB113a and also has the 1,
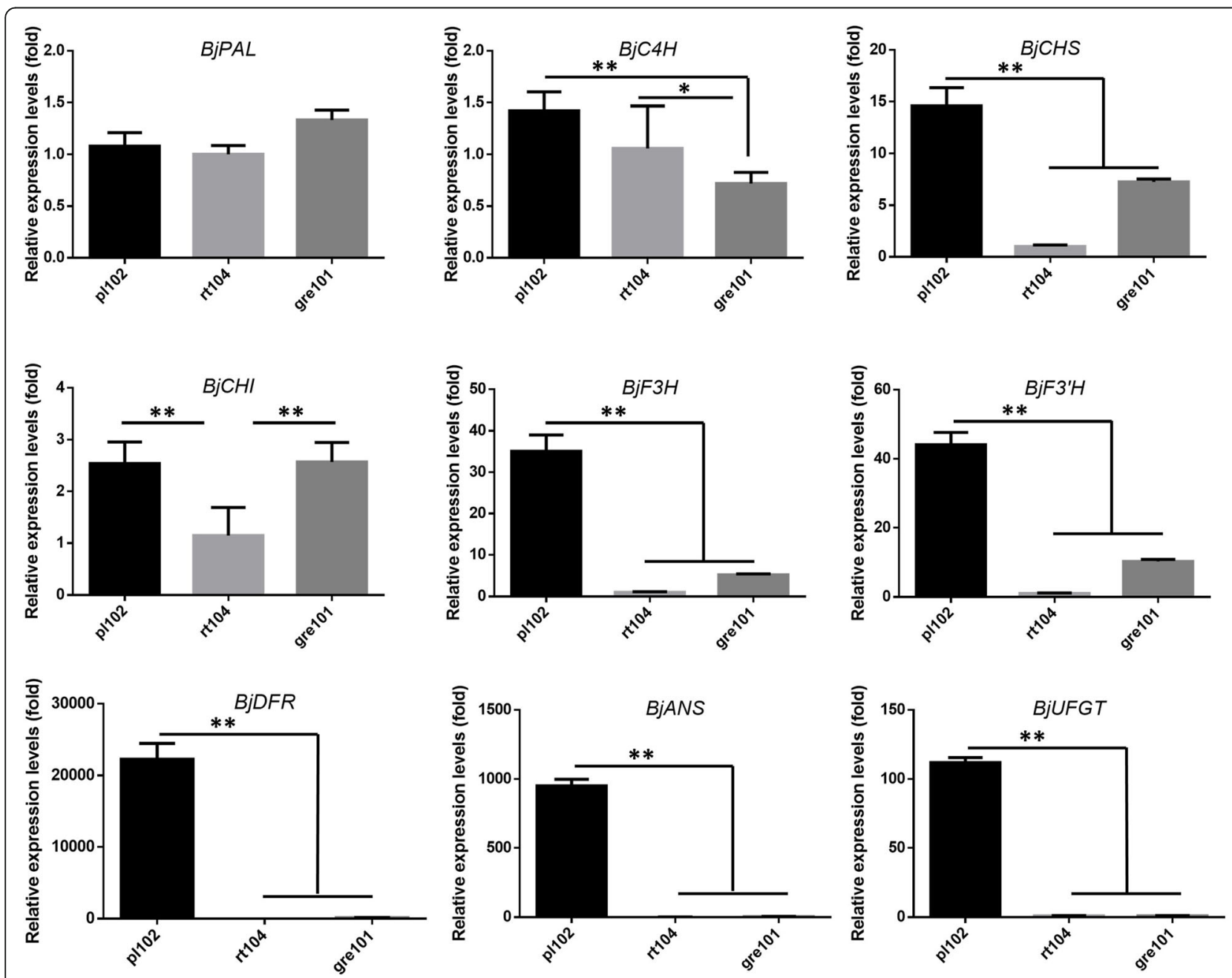

Fig. 7 The relative expression of nine anthocyanin biosynthesis genes in three Brassica juncea cultivars pl102, rt104 and gre101. Statistical analyzes were conducted by Student's t test, ${ }^{*} P<0.05 ;{ }^{*} P<0.01$ 


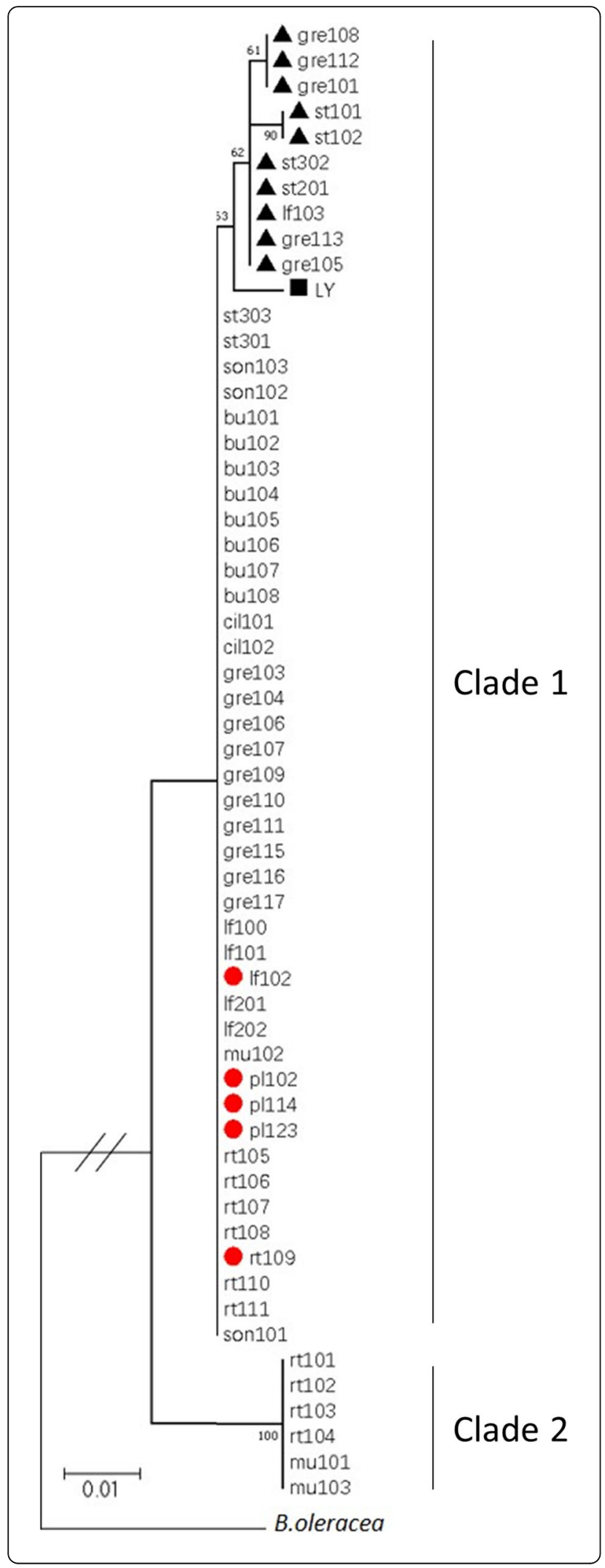

Fig. 8 The neighbor-joining tree of the sequences of the BjMYB113 alleles in 58 mustard accessions. The sequences of BjMYB113 ortholog in B. oleracea was used as an outgroup. Red circle represents purple phenotypes, and others are all green phenotypes. Black triangle represents the 1,033-bp insertion in the BjMYB113 gene in green mustards. Black rectangular represents the 1,268-bp insertion in the BjMYB113 gene in green mustards

033-bp insertion. The fourth sequence (five cultivars) has the same promoter sequence as BjMYB113a and with the 1,033-bp insertion. The fifth sequence (one cultivar LY) varies a 38-bp insertion in the promoter sequence and has the 1,268-bp insertion in intron 1 . Therefore, all promoter sequences from Clade 1 are most likely activated ones (see above). Surprisingly, of the 52 cultivars in Clade 1, 36 have green leaves but they do not have the large insertions in either intron 1 or in its 3' downstream. Three genotypes of 36 green cultivars were randomly chosen as representatives to investigate the expression level of BjMYB113. The expression level of $B j M Y B 113$ from three green cultivars were significantly lower than $B j M Y B 113 a$ from purple cultivar, suggesting that there might be other mutation events leading to the inactivation of the BjMYB113 gene or loss-of-function mutations in other genes of the anthocyanin biosynthesis pathway (Fig. S3).

\section{Discussion}

MYB plays a central role in color variation in plant species In this study, we showed that the BjMYB113 gene was responsible for the color variation in mustard, and point mutations and small InDels in the promoter region accounted for its activation and accumulation of the anthocyanins. Color polymorphisms might be caused by any genes in the anthocyanin biosynthesis pathways. However, the most frequent causal genes for natural color variations were MYB-encoding genes. For example, the RLL2 gene which encodes an R2R3-MYB transcription factor, regulates multiple genes from the anthocyanin biosynthesis pathways and promote the high-level accumulation of anthocyanins in lettuce leaves [29]. In $B$. oleracea species, the BoMYB2 gene has been activated independently for at least three times [31, 32]. The blood orange arose by insertion of a Copia-like retrotransposon to a MYB gene Ruby [26]. The OsC1 mutations in the coding region vary degrees of apiculus coloration in rice [36].

While wild species in natural populations have green leaves, many horticultural cultivars have red/purple leaves, such as red leaf plum, purple lettuce and purple mustard. With the assumption that their wild ancestors had green leaves, the red or purple cultivars arose from mutations and artificially selected and maintained during domestication or breeding. We showed in this study that 
the BjMYB113 gene was activated due to the accumulated point mutations and/or InDels. It will be interestingly to investigate which point mutation(s)/InDel(s) played the critical role in its activation.

In this study, we also found a mutation event that inactivated the BjMYB113 gene, a large insertion in its downstream. BLASTN search of the inserted sequences showed that it had a high copy number in the genome of mustard. The inserted sequences do not show similarity to known transposable elements, and the sequences per se do not show a typical transposon structure such as target site duplication (TSD) or terminal inverted repeats (TIR).

The mechanism for the inactivation of the BjMYB113 gene by the insertion remains unknown. One possibility is that the insertion may result in methylation in this region to downregulate its expression. We investigated cytosine methylation status in this region, but no significant difference in methylation ratio was found between $B j M Y B 113 a$ and BjMYB113c (Fig. S4). Another possibility is that the insertion disrupts an enhancer motif of the gene. It is also possible that the insertion might have altered the chromatin structure and consequently downregulates gene expression in its vicinity.

Our sequence analysis suggested that at least 36 cultivars with green leaves have the activated promoter of the BjMYB113a allele from the purple cultivar, but they do not have the 1,033-bp insertion in its 3' downstream or 1,268-bp insertion in intron 1 that could silence the gene. We sequenced the entire BjMYB113 gene from some cultivars, but no sequences variations were identified. Therefore, these green phenotypes were most likely caused by loss-of-function mutations of other genes in the anthocyanin biosynthesis pathway rather than the BjMYB113 gene.

In a recent report, a 1,268-bp insertion in the first intron suppressed the BjMYB113 gene, leading to a low anthocyanin accumulation [3]. The only difference between the promoter sequences of the BjMYB113 gene from that cultivar LY and the BjMYB113a allele is a 38bp insertion, and it is still unclear whether the low expression of BjMYB113 gene in the cultivar is due to the 38-bp insertion in its promoter or the insertion of the 1 , 268-bp in its intron 1 (Fig. 4).

\section{Different mutation events activating genes associated with anthocyanin biosynthesis}

Many mutation events may activate genes associated with anthocyanin biosynthesis, including point mutations, insertions of transposable elements, gene duplications, and InDels in promoter region. Insertions of a CACTA-like transposon and a harbinger transposon activated BoMYB2, leading to anthocyanin accumulation in purple kale and purple cauliflower, respectively [31,
32]. A non-LTR retrotransposon activated $\mathrm{CaAn2}$ in purple Capsicum annuum [37]. A Copia-like retrotransposon activated Ruby leading to blood orange in Citrus [26]. Gene duplications and mutations in promoter region activated the RLL2 gene leading to activated anthocyanin biosynthesis in lettuce [29]. The promoter variation of two $B$ alleles determine distinct tissue specificities of anthocyanin production in maize [38].

\section{BSA in combination with RNA-seq is an efficient method to map and clone causal genes}

BSR-seq, an efficient and economic method, has been widely applied to identify genes that control important traits in many crops, including wheat [39], cabbage [40], watermelon [41], rice [42], cauliflower [43], and Chinese kale [44]. In this study, the $M P L$ gene was also cloned through the BSR-seq analysis of $F_{2}$ segregating populations. RNA-seq may not detect as many SNPs as DNAsEq. However, in most cases, the number of SNPs identified by RNA-seq is enough for genetic analysis. Large difference of allele frequency of a SNP between two extreme pools provides direct evidence for the linkage between the SNP and a causal gene. The RNA-seq of the two pools also provides information on differentially expressed genes (DEGs). The DEGs, if located in the candidate region, might facilitate the identification of candidate gene. The GO analysis of the DEGs between the two extreme pools may also suggest the pathways that are associated with the trait of interest. In this study, the causal gene for the color variation in mustard, $B j M Y B 113$, showed expression difference between the two pools. Furthermore, several genes in the anthocyanin biosynthesis pathway were up-regulated in the purple pool compared with the green pool of the $F_{2}$ segregating population. All these information greatly helped us pinpointing the BjMYB113 gene as the causal gene and understanding the pathway involved for the color variation in mustard.

\section{Conclusions}

We genetically cloned an R2R3-MYB coding gene controlling purple leaves in mustard. BjMYB113 was activated due to promoter variations, leading to the high expression of genes in the anthocyanin biosynthesis pathway and the high accumulation of anthocyanin in the purple cultivar. However, a large insertion in its 3'region or in its first intron compromised the high expression in the active allele leading to green color. These results indicated that BjMYB113 transcription factor, as the important member of the MBW ternary complex, has experienced both gain-of-function and loss-offunction mutations during artificial selection and domestication. Our results contribute to a better understanding of the genetics and evolution of the BjMYB113 gene 
controlling purple leaves and provide useful information for further breeding programs of mustard, which will be of broad interest to biologists.

\section{Methods}

Plant materials and genetic segregating populations

Purple mustard cultivar pl102 and green cultivars rt104 and gre101 were chosen to study the genetics of purple color leaves. These three mustard cultivars are brown mustard (Brassica juncea). Purple cultivar pl102 was crossed with green cultivars rt104 and gre101, respectively, to develop two $F_{2}$ segregating populations. These $\mathrm{F}_{2}$ segregating populations along with its parents were acquired from professor Hanhui Kuang's lab (College of Horticulture and Forestry Sciences, Huazhong Agricultural University) in this study. Parental seed lines were originally acquired at National Center for Vegetable Improvement (Central China). Seeds were germinated and seedings grew in a greenhouse under $16 / 8 \mathrm{~h}$ photoperiod at $25{ }^{\circ} \mathrm{C} \pm 2{ }^{\circ} \mathrm{C}$. All plants were planted to the field on campus of Huazhong Agriculture University, Wuhan, China.

\section{Analysis of total anthocyanin contents}

Method of total anthocyanin content analysis followed the guidelines and protocols described previously [45]. For anthocyanin extraction, $0.1 \mathrm{~g}$ tissues of each sample were incubated in $300 \mu \mathrm{L}$ of extraction buffer (methanol containing $1 \% \mathrm{HCl}$ ) overnight at $4{ }^{\circ} \mathrm{C}$ in the dark. After extraction, $200 \mu \mathrm{L}$ of water and $200 \mu \mathrm{L}$ of chloroform were added to each sample, and absorbances were read at 530 and $657 \mathrm{~nm}$. The quantity of anthocyanin was determined by calculating absorbance at $530 \mathrm{~nm}$ (A530) $0.33 \mathrm{~A} 657$, and each sample was extracted and measured in three independent experiments.

\section{Flavonoid metabolite profiling}

The relative quantities of flavonoid metabolites in Brassica juncea leaf samples were analyzed with a liquid chromatography-electrospray ionization-tandem mass spectrometry (LC-ESI-MS/MS) system by MetWare (Wuhan, China). The liquid chromatographyelectrospray ionization-tandem mass spectrometry (LC-ESI-MS/MS) system was used for the relative quantification of anthocyanin metabolites in Brassica juncea leaves samples. The protocols were described detailedly in Methods S1.

\section{Bulked Segregant Analysis and RNA-seq (BSR-seq)}

Bulked Segregant Analysis in combination with RNAseq (BSR-seq) was used to map genes controlling purple leaves [46]. A total of 20 purple individuals from an $F_{2}$ segregating population were mixed as the purple-pool, and 20 green individuals were mixed as the green-pool.
Total RNAs were extracted from the two pools using RNAiso plus (Takara, Japan). RNA-seq was performed on Illumina Hiseq2500 platform (Novogene, China), and approximately $5 \mathrm{~GB}$ clean data were obtained for each pool. The data were mapped to Brassica juncea reference genome [47], using the Bowtie software [48]. SNP callings were performed using SAMtools [49]. Lowquality SNPs with map quality value $<30$, reads depth $<$ $10 \times$ or base quality value $<20$ were excluded. The key parameter of $\Delta$ (SNP-index) was used to identify the target region for purple/green traits [50]. The $\Delta$ (SNPindex) was calculated by subtracting the SNP-index value of the green-pool from the SNP-index value of purple-pool. Cleaved amplified polymorphic sequence (CAPS) markers were designed in the candidate region, and were used to screen the population to fine map the casual gene. The primers used in map-based cloning were shown in Table S3. The RNA-seq data supporting the results of this study is available in the NCBI SRA (Sequence Read Archive, http://www.ncbi.nlm.nih.gov/ sra/) repository under BioProject PRJNA672814.

\section{RNA extraction and quantitative Real-Time reverse transcription polymerase chain reaction (qRT-PCR) analysis}

Total RNAs were extracted from leaves using RNAiso plus (Takara, Japan). The cDNA was synthesized using TransScript One-Step gDNA Removal and cDNA Synthesis SuperMix (TransScript, China) with Oligo-dT18 primer. qRT-PCR analysis followed the guidelines and protocols described previously [51]. All reactions were performed using the SYBR Premix $(5.0 \mu \mathrm{L}$ of $2 \times$ SYBR Premix Go Taq II, $0.5 \mu \mathrm{L}$ of primers, $1.0 \mu \mathrm{L}$ of $\mathrm{cDNA}$, and $3.5 \mu \mathrm{L}$ of ddH2O). Melting curve analysis of qRTPCR samples revealed that there was only one product for each gene primer reaction. The PCR products were sequenced to confirm the specific amplification. A house-keeping gene $B j E F-1-\alpha^{37}$ was used as an internal standard in tissues. The relative expression levels were counted using the formula $2^{-\Delta \Delta \mathrm{Cq}}$ as described in BioRad protocol, and statistical differences were calculated using student's test. Three biological replications and three technical replications were performed in qRTPCR. The primers used in qRT-PCR analysis were shown in Table S3.

\section{Plasmid construction and plant transformation}

The full-length BjMYB113 cDNA was cloned into the pRI101-GFP vector with CaMV $35 \mathrm{~S}$ promoter to construct the overexpression vector. The full-length BjMYB113 gene sequence (including native promoter and 3'region sequence) was amplified and cloned into the pRI101-GFP to construct the complementary vector. Approximately $3 \mathrm{~kb}$ promoter region of three BjMYB113 
alleles were amplified and recombined into the pCAMBIA1301-GUS vector for checking GUS activity. All vectors were constructed using homologous recombination. Positive plasmids were verified by sequencing and then transformed into Agrobacterium tumefaciens GV3101 using thermal stimulation method. The vectors were transformed into Arabidopsis using floral-dip method [52]. All primers used for vector construction were shown in Table S3.

\section{Promoter activity analysis}

The quantitative GUS activity was measured using the Lu's methods with slight modification [53]. GUS activity was detected in 1-month-old Arabidopsis leaf tissues $(10 \mathrm{mg})$ from three independent transgenic lines and six individuals in each line. Total proteins were extracted using $300 \mu \mathrm{L}$ GUS extraction buffer $(50 \mathrm{mM}$ phosphate buffer, pH 7.0; 10 mM EDTA, pH 8.0; 0.1\% Sodium Dodecvl Sulfate; $10 \mathrm{mM} \beta$-mercaptoethanol). BCA Protein Assay (Beyotime Biotechnology, China) was used to measure the protein concentrations. Extraction $(100 \mu \mathrm{L})$ was added to $900 \mu \mathrm{L}$ GUS extraction buffer containing 1 $\mathrm{mM}$ 4-methylumbelliferyl glucuronide (MUG, Sigma) and incubated at $37{ }^{\circ} \mathrm{C}$. The $900 \mu \mathrm{L}$ stop solution $(1 \mathrm{M}$ Sodium Carbonate) immediately added into $100 \mu \mathrm{L}$ the above reaction mixture and $60 \mathrm{~min}$ later, respectively. Fluorescence of 4-methylumbelliferone (MU) was monitored using Tecan Infnite ${ }^{\mathrm{Tm}}$ at $455 \mathrm{~nm}$ emission and 365 $\mathrm{nm}$ excitation. GUS activity was expressed as $\mu$ moles 4methylumbelliferone (MU) $\mathrm{min}^{-1} \mathrm{mg}^{-1}$ protein.

\section{Sequence analysis and neighbor-joining tree}

The promoter sequence of BjMYB113 alleles was analyzed using PlantCARE (http://bioinformatics.psb.ugent. be/webtools/plantcare/html/).

Sequence alignments were conducted using Muscle program and manually adjusted in GeneDoc (http:// www.nrbsc.org/gfx/genedoc/). The neighbor-joining tree was constructed using MEGA 7.0 [54] and bootstrap values were calculated using 1,000 times.

The gene sequences (BjMYB113a, -b, and -c) supporting the results of this study are available in the NCBI GenBank (https://www.ncbi.nlm.nih.gov/ genbank/) under accession number MW166171MW166173 of BankIt2394807. The primers used in gene sequences analysis were shown in Table S3.

\section{Methylation sensitive digestion}

Method of methylation sensitive digestion followed the guidelines and protocols described previously [55]. Genomic DNA was extracted from purple mustard cultivar pl102 and green cultivar gre101. The quality and integrity of extracted genomic DNA was evaluated by spectrophotometric analysis using NanoDrop (ThermoFisher
Scientific, U.S.A.). Genomic DNA was digested by the methylation-sensitive endonuclease McrBC according to the manufacturer's instructions (New England Biolab Inc., U.S.A.). Then, qRT-PCR analysis was performed [51]. Each sample was measured in three independent experiments. The mean $C_{T}$ values were used to calculate $\Delta \mathrm{C}_{\mathrm{T}}$ as follows: $\Delta \mathrm{C}_{\mathrm{T}}=\left[\mathrm{C}_{\mathrm{T}}\right.$ (McrBC treatment) $\mathrm{C}_{\mathrm{T}}($ Control)] and the methylation percentage was calculated as methylation $\%=100-\left(100 \times 2^{-\Delta \mathrm{C}}\right)$.

\section{Abbreviations}

ANS: Anthocyanidin synthase; BSR-seg: Bulked Segregant Analysis and RNAseq; CaMV: Cauliflower mosaic virus; CAPS: Cleaved amplified polymorphic sequence; CHI: Chalcone isomerase; CHS: Chalcone synthase;

DEGs: Differentially expressed genes; DFR: Dihydroflavonol 4-reductase; F3H: Flavanone 3-hydroxylase; GO: Gene Ontology; InDels: Insertions and Deletions; LC-ESI-MS: Liquid Chromatography-Electrospray lonization-Mass Spectrometry; MPL: Mustard Purple Leaves; MU: 4-methylumbelliferone; MUG: 4-methylumbelliferyl glucuronide; ORFs: Open reading frames; PAL: Phenylalanine-ammonia lyase; qRT-PCR: Quantitative Real-Time reverse transcription polymerase chain reaction; ROS: Reactive oxygen species; SNPs: Single nucleotide polymorphisms; TIR: Terminal inverted repeats; TSD: Target site duplication

\section{Supplementary Information}

The online version contains supplementary material available at https://doi. org/10.1186/s12870-021-03084-5.

Additional file 1: Figure S1. Map-based cloning of the MPL gene in $F_{2}$ population of pl102 $\times$ gre101.

Additional file 2: Figure S2. The alignment of protein sequences of BjMYB113 and its most similar R2R3-MYB transcription factors homologous genes.

Additional file 3: Figure S3. The relative expression of BjMYB113 of purple cultivar pl102 and three green cultivars.

Additional file 4: Figure S4. Analysis of the methylation status in the $3^{\prime}$ regions of BjMYB113a and BjMYB113C.

Additional file 5: Table S1. Different types of anthocyanin and their concentrations in purple and green cultivars with three biological replications.

Additional file 6: Table S2. The Chi-square (x2) of two purple/green segregating populations.

Additional file 7: Table S3. The primers used in this research.

Additional file 8: Method S1. Anthocyanin metabolite profiling.

\section{Acknowledgements}

We thank Dr. Changchun Yu (Huazhong Agricultural University) for the vector pRI101-GFP and pCAMBIA1301-GUS, Dr. Weiyi Zhang (Huazhong Agricultural University) for technique support of bioinformatics, Dr. Hanhui Kuang (Huazhong Agricultural University) for plant materials and helpful suggestions on this work.

\section{Authors' contributions}

J.C. designed the project. G.A. performed the experiments. G.A. wrote the manuscript with the helps from J.C. The author(s) read and approved the final manuscript.

\section{Funding}

This work was supported by grants from the National Key Research and Development Program of China [2018YFD1000800], and the Fundamental Research Funds for the Central Universities [2662018PY070]. All funders were not involved in the design of the study, data analysis, and writing the manuscript. 


\section{Availability of data and materials}

The data supporting the result of this study are within the paper and its additional files. All sequencing datasets are deposited in the National Centre for Biotechnology Information (NCBI) under the BioProject ID PRJNA672814 with the Sequence Read Achieve (SRA) accession SRR12919405-SRR12919408. The gene sequences (BjMYB113a, $-b$, and $-c$ ) supporting the results of this study are available in the NCBI GenBank (https://www.ncbi.nlm.nih.gov/ genbank/) under accession number MW166171 - MW166173 of Banklt2394807.

\section{Declarations}

Ethics approval and consent to participate

Not applicable.

\section{Consent for publication}

Not applicable.

\section{Competing interests}

The authors declare no conflict of interests.

Received: 12 January 2021 Accepted: 4 June 2021

Published online: 29 June 2021

\section{References}

1. Xie Q, Hu Z, Zhang Y, Tian S, Wang Z, Zhao Z, Yang Y, Chen G Accumulation and molecular regulation of anthocyanin in purple tumorous stem mustard (Brassica juncea var. tumida Tsen et Lee). J Agric Food Chem. 2014;62(31):7813-21.

2. Liu X, Lu Y, Yuan Y, Liu S, Guan C, Chen S, Liu Z. De novo transcriptome of Brassica juncea seed coat and identification of genes for the biosynthesis of flavonoids. PLoS One. 2013;8(8):e71110.

3. Heng S, Cheng Q, Zhang T, Liu X, Huang H, Yao P, Liu Z, Wan Z, Fu T. Finemapping of the BjPur gene for purple leaf color in Brassica juncea. Theor Appl Genet 2020: https://doi.org/10.1007/s00122-00020-03634-00129.

4. Kong J-M, Chia L-S, Goh N-K, Chia T-F, Brouillard R. Analysis and biological activities of anthocyanins. Phytochemistry. 2003:64(5):923-33.

5. Stuurman J, Hoballah ME, Broger L, Moore J, Basten C, Kuhlemeier C. Dissection of floral pollination syndromes in Petunia. Genetics. 2004;168(3): 1585-99.

6. Neill SO, Gould KS. Anthocyanins in leaves: light attenuators or antioxidants? Funct Plant Biol. 2003;30(8):865-73.

7. Gould KS. Nature's swiss army knife: the diverse protective roles of anthocyanins in leaves. J Biomed Biotechnol. 2004;2004(5):314-20.

8. Albert NW, Lewis DH, Zhang H, Irving $\sqcup$, Jameson PE, Davies KM. Lightinduced vegetative anthocyanin pigmentation in Petunia. J Exp Bot. 2009; 60(7):2191-202.

9. Olsen KM, Slimestad R, Lea US, Brede C, Lovdal T, Ruoff P, Verheul M, Lillo C. Temperature and nitrogen effects on regulators and products of the flavonoid pathway: experimental and kinetic model studies. Plant Cell Environ. 2009;32(3):286-99.

10. Zhang Y, Butelli E, De Stefano R, Schoonbeek HJ, Magusin A, Pagliarani C, Wellner N, Hill L, Orzaez D, Granell A, et al. Anthocyanins double the shelf life of tomatoes by delaying overripening and reducing susceptibility to gray mold. Curr Biol. 2013;23(12):1094-100.

11. Cao X, Qiu Z, Wang X, Van Giang T, Liu X, Wang J, Wang X, Gao J, Guo Y, Du $Y$, et al. A putative $R 3$ MYB repressor is the candidate gene underlying atroviolacium, a locus for anthocyanin pigmentation in tomato fruit. J Exp Bot. 2017:68(21-22):5745-58.

12. Wang LS, Stoner GD. Anthocyanins and their role in cancer prevention. Cancer Lett. 2008;269(2):281-90.

13. Zhang Y, Butelli E, Martin C. Engineering anthocyanin biosynthesis in plants. Curr Opin Plant Biol. 2014:19:81-90

14. Tsuda T, Horio F, Uchida K, Aoki H, Osawa T. Dietary cyanidin 3-O-beta-Dglucoside-rich purple corn color prevents obesity and ameliorates hyperglycemia in mice. J Nutr. 2003;133(7):2125-30.

15. Rasmussen SE, Frederiksen H, Krogholm KS, Poulsen L. Dietary proanthocyanidins: Occurrence, dietary intake, bioavailability, and protection against cardiovascular disease. Mol Nutr Food Res. 2005;49(2):159-74.

16. Butelli E, Titta L, Giorgio M, Mock HP, Matros A, Peterek S, Schijlen EGWM, Hall RD, Bovy AG, Luo J, et al. Enrichment of tomato fruit with health- promoting anthocyanins by expression of select transcription factors. Nat Biotechnol. 2008;26(11):1301-8.

17. Koes R, Verweij W, Quattrocchio F. Flavonoids: a colorful model for the regulation and evolution of biochemical pathways. Trends Plant Sci. 2005: 10(5):236-42.

18. Ramsay NA, Glover BJ. MYB-bHLH-WD40 protein complex and the evolution of cellular diversity. Trends Plant Sci. 2005;10(2):63-70.

19. Gonzalez A, Zhao M, Leavitt JM, Lloyd AM. Regulation of the anthocyanin biosynthetic pathway by the TTG1/bHLH/Myb transcriptional complex in Arabidopsis seedlings. Plant J. 2008;53(5):814-27.

20. Holton TA, Cornish EC. Genetics and biochemistry of anthocyanin biosynthesis. Plant Cell. 1995;7(7):1071-83.

21. Springob K, Nakajima J, Yamazaki M, Saito K. Recent advances in the biosynthesis and accumulation of anthocyanins. Nat Prod Rep. 2003;20(3): 288-303.

22. Zhou H, Lin-Wang K, Wang H, Gu C, Dare AP, Espley RV, He H, Allan AC, Han Y. Molecular genetics of blood-fleshed peach reveals activation of anthocyanin biosynthesis by NAC transcription factors. Plant J. 2015;82(1): 105-21.

23. Espley RV, Hellens RP, Putterill J, Stevenson DE, Kutty-Amma S, Allan AC. Red colouration in apple fruit is due to the activity of the MYB transcription factor, MdMYB10. Plant J. 2007:49(3):414-27.

24. Li C, Wu J, Hu KD, Wei SW, Sun HY, Hu LY, Han Z, Yao GF, Zhang H. PyWRKY26 and PybHLH3 cotargeted the PyMYB114 promoter to regulate anthocyanin biosynthesis and transport in red-skinned pears. Hortic Res. 2020;7:37

25. Walker AR, Lee E, Bogs J, McDavid DAJ, Thomas MR, Robinson SP. White grapes arose through the mutation of two similar and adjacent regulatory genes. Plant J. 2007:49(5):772-85.

26. Butelli E, Licciardello C, Zhang Y, Liu J, Mackay S, Bailey P, ReforgiatoRecupero G, Martin C. Retrotransposons control fruit-specific, colddependent accumulation of anthocyanins in blood oranges. Plant Cell. 2012;24(3):1242-55.

27. Quattrocchio F, Wing J, van der Woude K, Souer E, de Vetten N, Mol J, Koes R. Molecular analysis of the anthocyanin2 gene of petunia and its role in the evolution of flower color. Plant Cell. 1999;11(8):1433-44.

28. Sweeney MT, Thomson MJ, Pfeil BE, McCouch S. Caught red-handed: Rc encodes a basic helix-loop-helix protein conditioning red pericarp in rice. Plant Cell. 2006;18(2):283-94.

29. Su W, Tao R, Liu W, Yu C, Yue Z, He S, Lavelle D, Zhang W, Zhang L, An G, et al. Characterization of four polymorphic genes controlling red leaf colour in lettuce that have undergone disruptive selection since domestication. Plant Biotechnol J. 2020;18(2):479-90.

30. Zhang Y, Chen G, Dong T, Pan Y, Zhao Z, Tian S, Hu Z. Anthocyanin accumulation and transcriptional regulation of anthocyanin biosynthesis in purple bok choy (Brassica rapa var. chinensis). J Agric Food Chem. 2014; 62(51):12366-76.

31. Yan C, An G, Zhu T, Zhang W, Zhang L, Peng L, Chen J, Kuang H. Independent activation of the BOMYB2 gene leading to purple traits in Brassica oleracea. Theor Appl Genet. 2019;132(4):895-906.

32. Chiu LW, Zhou X, Burke S, Wu X, Prior RL, Li L. The purple cauliflower arises from activation of a MYB transcription factor. Plant Physiol. 2010;154(3): $1470-80$.

33. Li P, Chen B, Zhang G, Chen L, Dong Q, Wen J, Mysore KS, Zhao J. Regulation of anthocyanin and proanthocyanidin biosynthesis by Medicago truncatula bHLH transcription factor MtTR. New Phytol. 2016; 210(3):905-21.

34. Amato A, Cavallini E, Zenoni S, Finezzo L, Begheldo M, Ruperti B, Tornielli GB. A grapevine TTG2-Like WRKY transcription factor is involved in regulating vacuolar transport and flavonoid biosynthesis. Front Plant Sci. 2017;7:1979.

35. Peng XX, Hu YJ, Tang XK, Zhou PL, Deng XB, Wang HH, Guo ZJ. Constitutive expression of rice WRKY30 gene increases the endogenous jasmonic acid accumulation, $P R$ gene expression and resistance to fungal pathogens in rice. Planta. 2012;236(5):1485-98.

36. Saitoh K, Onishi K, Mikami I, Thidar K, Sano Y. Allelic diversification at the C (OsC1) locus of wild and cultivated rice: nucleotide changes associated with phenotypes. Genetics. 2004;168(2):997-1007.

37. Jung S, Venkatesh J, Kang MY, Kwon JK, Kang BC. A non-LTR retrotransposon activates anthocyanin biosynthesis by regulating a MYB transcription factor in Capsicum annuum. Plant Sci. 2019;287:110181. 
38. Radicella JP, Brown D, Tolar LA, Chandler VL. Allelic diversity of the maize $B$ regulatory gene: different leader and promoter sequences of two $B$ alleles determine distinct tissue specificities of anthocyanin production. Genes Dev. 1992:6(11):2152-64.

39. Li Y, Xiong H, Guo H, Zhou C, Xie Y, Zhao L, Gu J, Zhao S, Ding Y, Liu L. Identification of the vernalization gene VRN-B1 responsible for heading date variation by QTL mapping using a RIL population in wheat. BMC Plant Biol. 2020:20(1):331.

40. Li R, Hou Z, Gao L, Xiao D, Hou X, Zhang C, Yan J, Song L. Conjunctive analyses of BSA-Seq and BSR-Seq to reveal the molecular pathway of leafy head formation in Chinese cabbage. Plants (Basel). 2019;8(12):603.

41. Sun L, Zhang Y, Cui H, Zhang L, Sha T, Wang C, Fan C, Luan F, Wang X. Linkage mapping and comparative transcriptome analysis of firmness in watermelon (Citrullus lanatus). Front Plant Sci. 2020;11:831.

42. Gao J, Dai G, Zhou W, Liang H, Huang J, Qing D, Chen W, Wu H, Yang X, Li $D$, et al. Mapping and identifying a candidate gene Plr4, a recessive gene regulating purple leaf in rice, by using bulked segregant and transcriptome analysis with next-generation sequencing. Int J Mol Sci. 2019;20(18):4335.

43. Zhao Z, Sheng $X, Y u H$, Wang J, Shen $Y, G u$ H. Identification of candidate genes involved in curd riceyness in cauliflower. Int J Mol Sci. 2020;21(6):1999.

44. Tang QW, Tian MY, An GH, Zhang WY, Chen JJ, Yan CH. Rapid identification of the purple stem $\left(P_{s}\right)$ gene of Chinese kale (Brassica oleracea var. alboglabra) in a segregation distortion population by bulked segregant analysis and RNA sequencing. Mol Breed. 2017:37(12):153.

45. Shin J, Park E, Choi G. PIF3 regulates anthocyanin biosynthesis in an HY5dependent manner with both factors directly binding anthocyanin biosynthetic gene promoters in Arabidopsis. Plant J. 2007;49(6):981-94.

46. Liu S, Yeh CT, Tang HM, Nettleton D, Schnable PS. Gene mapping via bulked segregant RNA-Seq (BSR-Seq). PLoS One. 2012;7(5):e36406.

47. Yang J, Liu D, Wang X, Ji C, Cheng F, Liu B, Hu Z, Chen S, Pental D, Ju Y, et al. The genome sequence of allopolyploid Brassica juncea and analysis of differential homoeolog gene expression influencing selection. Nat Genet. 2016;48(10):1225-32

48. Langmead B, Trapnell C, Pop M, Salzberg SL. Ultrafast and memory-efficient alignment of short DNA sequences to the human genome. Genome Biol. 2009;10(3):R25.

49. Li H, Handsaker B, Wysoker A, Fennell T, Ruan J, Homer N, Marth G, Abecasis G, Durbin R. Genome Project Data Processing S: The sequence alignment/ map format and SAMtools. Bioinformatics. 2009;25(16):2078-9.

50. Takagi H, Abe A, Yoshida K, Kosugi S, Natsume S, Mitsuoka C, Uemura A, Utsushi H, Tamiru M, Takuno S, et al. QTL-seq: rapid mapping of quantitative trait loci in rice by whole genome resequencing of DNA from two bulked populations. Plant J. 2013;74(1):174-83.

51. Udvardi MK, Czechowski T, Scheible WR. Eleven golden rules of quantitative RT-PCR. Plant Cell. 2008;20(7):1736-7.

52. Clough SJ, Bent AF. Floral dip_ a simplified method for Agrobacteriummediated transformation of Arabidopsis thaliana. Plant J. 1998;16(6):735-43.

53. Lu S, Zhang $Y$, Zheng $X$, Zhu $K$, Xu Q, Deng X. Isolation and functional characterization of a lycopene beta-cyclase gene promoter from citrus. Front Plant Sci. 2016;7:1367.

54. Kumar S, Stecher G, Tamura K. MEGA7: Molecular Evolutionary Genetics Analysis Version 7.0 for Bigger Datasets. Mol Biol Evol. 2016;33(7):1870-4.

55. Sicilia A, Scialo E, Puglisi I, Lo Piero AR. Anthocyanin biosynthesis and DNA methylation dynamics in sweet orange fruit [Citrus sinensis L. (Osbeck)] under cold stress. J Agric Food Chem. 2020;68(26):7024-31.

\section{Publisher's Note}

Springer Nature remains neutral with regard to jurisdictional claims in published maps and institutional affiliations.

Ready to submit your research? Choose BMC and benefit from:
- fast, convenient online submission
- thorough peer review by experienced researchers in your field
- rapid publication on acceptance
- support for research data, including large and complex data types
- gold Open Access which fosters wider collaboration and increased citations
- maximum visibility for your research: over 100M website views per year
At BMC, research is always in progress.
Learn more biomedcentral.com/submissions

